\title{
The Standard Model Fermion Spectrum From Complex Projective Spaces
}

\author{
Brian P. Dolan and C. Nash \\ Dept. of Mathematical Physics, NUI, Maynooth, Ireland \\ and \\ School of Theoretical Physics, Dublin Institute for Advanced Studies, \\ 10 Burlington Rd., Dublin 8, Ireland \\ bdolan@thphys.may.ie, cnash@thphys.may.ie
}

\begin{abstract}
It is shown that the quarks and leptons of the standard model, including a right-handed neutrino, can be obtained by gauging the holonomy groups of complex projective spaces of complex dimensions two and three. The spectrum emerges as chiral zero modes of the Dirac operator coupled to gauge fields and the demonstration involves an index theorem analysis on a general complex projective space in the presence of topologically non-trivial $S U(n) \times U(1)$ gauge fields. The construction may have applications in type IIA string theory and non-commutative geometry.
\end{abstract}

Keywords: Field Theories in Higher Dimensions, Differential and Algebraic Geometry, Non-Commutative Geometry. 


\section{Contents}

1. Introduction 1

2. The Particle Spectrum From $\mathrm{CP}^{n}$

3. Conclusions 6

A. The Index Theorem For CP ${ }^{n}$

\section{Introduction}

It has been a long standing goal of theoretical particle physics to unify space-time symmetries with the internal $S U(3) \times S U(2) \times U(1)$ gauge symmetry of the standard model. Most current research in this direction relies on string theory in higher dimensions, hoping to derive grand unified theories as low energy limits of the full theory, and a crucial aspect of this programme is the rôle of compact internal spaces. Compact internal spaces were first introduced into physics in Kaluza-Klein models where a coset space $G / H$ with isometry group $G$ and holonomy group $H$ can give rise to a gauge group $G$ in 4-dimensional space-time. A pure Kaluza-Klein approach was largely abandoned in the $80 \mathrm{~s}$ due in part to the realisation that it was difficult, if not impossible, to obtain chiral Fermions this way [1]. In this paper we take a different approach to internal coset spaces, focusing on the holonomy group $H$ rather than $G$. We shall show that a single generation of the standard model spectrum, including a right handed neutrino, can arise from the complex projective spaces $\mathbf{C P}^{2}$ and $\mathbf{C P}^{3}$.

The approach adopted here has the attractive feature that it is somewhat more in tune with the spirit of general relativity than standard Kaluza-Klein theory. In the standard approach one assumes that the coset space has a specific metric which has isometry group $G$, which is somewhat contrary to the philosophy general relativity where a particular metric is merely one solution of Einstein's field equations and there may be many others with smaller isometries, indeed a generic solution has none. In the construction used here it is the holonomy group that is important and for a complex manifold with real dimension $2 n$ this group is generically $U(n)$. For 4-dimensional space time equipped with a Lorentzian metric the holonomy group is generically $S O(1,3)$ and general relativity can be viewed as a gauge theory of tangent space rotations using this group. Incorporating spinors into general relativity requires 
gauging the double cover of $S O(1,3)$, namely $\operatorname{Spin}(1,3) \cong S l(2, \mathbf{C})$. If a compact complex internal space with real dimension $2 n$ is added the extra holonomy group is generically $U(n)$ and so it seems quite natural to gauge $S U(n) \times U(1)$. Taking two internal spaces with $n=2$ and $n=3$ would give $S U(3) \times S U(2) \times U(1) \times U(1)$ which is not quite what is required, but is close.

To see how the standard model spectrum might arise in this way consider first a Dirac spinor on a general compact complex manifold of real dimension 4. This has 4 components and decomposes as

$$
(\mathbf{2}, \mathbf{1})+(\mathbf{1}, \mathbf{2}) \longrightarrow \mathbf{2}_{0}+\left(\mathbf{1}_{1}+\mathbf{1}_{-1}\right)
$$

under $\operatorname{Spin}(4) \cong S U(2) \times S U(2) \rightarrow S U(2) \times U(1)$. Shifting the $U(1)$ charge by -1 and rescaling the result by $1 / 2$ gives

$$
\mathbf{2}_{-1 / 2}+\left(\mathbf{1}_{0}+\mathbf{1}_{-1}\right) .
$$

On the other hand a compact complex manifold of real dimension 6 has 8 component Dirac spinors which decompose as

$$
\mathbf{4}+\overline{\mathbf{4}} \longrightarrow\left(\mathbf{3}_{1}+\mathbf{1}_{-3}\right)+\left(\overline{\mathbf{3}}_{-1}+\mathbf{1}_{3}\right)
$$

under $\operatorname{Spin}(6) \cong S U(4) \rightarrow S U(3) \times U(1)$. Taking a single chiral spinor in the 4 of $S U(4)$, shifting the $U(1)$ charge by 3 and rescaling the result by $1 / 6$, gives

$$
\left(\mathbf{3}_{2 / 3}+\mathbf{1}_{0}\right) .
$$

A single generation of the standard model spectrum, including a right-handed neutrino, can be obtained by tensoring (1.2) with (1.4) and simply adding the $U(1)$ charges. These group theory decompositions are valid on any complex manifolds of real dimension 4 and 6 respectively and the shift in the $U(1)$ charges may be achieved by coupling the Fermions to $U(1)$ gauge fields with appropriate topological (monopole) charges. Mathematically this requires tensoring the spin bundle with an appropriate line bundle, which may or may not be possible on any given manifold depending on whether or not appropriate line bundles exist. It is shown in section 2 that for $\mathbf{C P}^{2}$ and $\mathbf{C} \mathbf{P}^{3}$ the required line bundles do indeed exist (indeed for $\mathbf{C P}^{2}$ spinors cannot even be defined unless such a line bundle is introduced). However obtaining the standard model spectrum requires introducing some natural higher rank bundles as well. Furthermore, an index theorem analysis shows that the representations (1.2) and (1.4) can be realised as zero modes of the Dirac operator with precisely the correct handedness for one generation of the standard model, including a right-handed neutrino. This is a topological statement, it is completely independent of the choice of metric on these spaces.

Of course there is the question of generations - the construction presented here only produces a single generation of the standard model. This point is elaborated 
on in the concluding section 3, along with some suggestions of how the construction might fit into viable models such as type IIA superstring theory and non-commutative geometry. Details of the index theorem on $\mathbf{C P}^{n}$, required for the analysis in the text, are contained in an appendix, where closed form expressions for the index of the Dirac operator coupled to topologically non-trivial $S U(n) \times U(1)$ gauge fields are derived.

\section{The Particle Spectrum From $\mathrm{CP}^{n}$}

In this section it will be argued that the spectrum of a single generation of the standard model can be obtained from $\mathbf{C P}^{2} \times \mathbf{C P}^{3}$ by gauging the holonomy group, $U(2) \times U(3)$, with the two $U(1)$ factors identified in one particular combination. This generalises the case of $\mathbf{C P}^{2}$ which gives the electroweak sector [2].

The analysis is based on the index theorem for the Dirac operator on

$$
\mathbf{C P}^{n} \cong \frac{S U(n+1)}{U(n)}
$$

derived in the appendix. We are interested in zero modes of the Dirac operator, for Fermions which are either singlets or transform under the fundamental $\mathbf{n}$ representation of $S U(n)$, when topologically non-trivial gauge fields are present $\left(\mathbf{C P}^{n}\right.$ analogues of the monopole field on $S^{2}$ and the $S U(2)$ BPST instanton on $S^{4}$ ). The gauge group is taken to be the holonomy group $U(n)$ of $\mathbf{C P}^{n}$. The index is the difference between the number of positive chirality zero modes of the Dirac operator and the number of negative chirality zero modes and, for convenience, the important formulae from the appendix are reproduced here. For a singlet with $U(1)$ charge $Y_{(n)}=q$, where $q$ is an integer, the index is

$$
\nu_{q}=\frac{1}{n !}(q+1) \cdots(q+n), q \in \mathbf{Z}
$$

corresponding to a line bundle over $\mathbf{C P}^{n}$ with gauge group $U(1)$. A Fermion in the fundamental representation of $S U(n)$, with a $U(1)$ charge $Y_{(n)}=q+\frac{1}{n}$, has index

$$
\nu_{q, \mathbf{n}}=\frac{(q+1) \cdots(q+(n-1))(q+n+1)}{(n-1) !} .
$$

We are free to rescale the $U(1)$ charges differently for different values of $n$, but for a given $n$ the ratio of the singlet charge $q$ to the fundamental charge $q+\frac{1}{n}$ is fixed.

The simplest case is $\mathbf{C P}^{1} \cong S^{2}$, where the holonomy group is $U(1)$. Introducing $U(1)$ gauge fields and Fermions with charge $q$ there are $\nu_{q}=q+1$ zero modes of the Dirac operator. The case $q=-1$ gives no unpaired zero modes-generically there are none at all but even when zero modes exist left and right-handed particles with charge -1 occur in pairs, like an electron in QED. For $q \neq-1$ the theory is necessary chiral. For example choosing the convention that positive $\nu$ corresponds to 
right-handed spinors in 4-dimensions, $q=-2$ would be interpreted as a left-handed particle with hypercharge -2 . However there is no place in this construction for a right-handed particle with the same charge as this would require $q=-2$ and $\nu>0$. This is necessarily a chiral theory for $q \neq-1$, and would generate a lethal gauge anomaly in 4-dimensions.

To introduce quarks we turn to $\mathbf{C} \mathbf{P}^{3}$ - since the holonomy group of $\mathbf{C P}^{3}$ is $U(3)$ quarks can be introduced as triplets. For example an $S U(3)$ triplet with $q=-3$ gives $\nu_{-3,3}=1$ and has $U(1)$ charge $Y_{(3)}=-8 / 3$. Since the overall normalisation of the charge is at our disposal, this could represent either:

$$
\text { a right handed d-quark (rescale charge by } 1 / 8), \quad d_{\mathbf{R}}=\mathbf{3}_{-1 / 3},
$$

or

$$
\text { a right-handed u-quark (rescale charge by }-1 / 4), \quad u_{\mathbf{R}}=\mathbf{3}_{2 / 3} \text {. }
$$

Either possibility forces us to interpret positive chirality as corresponding to righthanded Fermions in 4-dimensions in order to match the standard model spectrum. It is instructive to examine the complex conjugate representations in order to understand the CPT conjugate of the spectra. In the appendix it is shown that under complex conjugation of the bundles over $\mathbf{C P}^{n}$ the $U(1)$ charges transform as $Y_{(n)} \rightarrow \bar{Y}_{(n)}=-Y_{(n)}-(n+1)$ and the index as $\nu \rightarrow \bar{\nu}=(-1)^{n} \nu$. So for the examples (2.4) and (2.5) above, with just a single $S U(3)$ triplet on $\mathbf{C P}^{3}$ with $Y_{(3)}=-8 / 3$, $\bar{Y}_{(3)}=-4 / 3$ and complex conjugation flips the chirality so it maps

$$
d_{\mathbf{R}}=\mathbf{3}_{-1 / 3} \longrightarrow \overline{\mathbf{3}}_{-1 / 6}
$$

and

$$
u_{\mathbf{R}}=\mathbf{3}_{2 / 3} \longrightarrow \overline{\mathbf{3}}_{1 / 3}=(\bar{d})_{\mathbf{L}}
$$

If we start with a right-handed $u$-quark complex conjugation forces us to introduce the left-handed anti- $d$ while starting with a right-handed $d$-quark complex conjugation forces the introduction of a state that has no place in the standard model. This nicely illustrates the kind of constraints that CPT can place on the possible choices.

For weak interactions we bring in $\mathbf{C P}^{2}$ with holonomy group $U(2)$. An index theorem analysis then allows us to obtain a single generation of the electroweak sector, including a right-handed neutrino. This is achieved by taking the following three representations:

- An $S U(2)$ singlet with $q=0$ giving zero charge and index $\nu_{0}=+1$;

- A second singlet with $q=-3$ giving charge $Y_{(2)}=-3$ and index $\nu_{-3}=+1$;

- An $S U(2)$ doublet with $q=-2$ giving charge $Y_{(2)}=-3 / 2$ and $\nu_{-2,2}=-1$. 
Interpreting positive $\nu$ as giving right-handed spinors, and rescaling the charge by $1 / 3$, this results in a single generation of particles of the electroweak sector of the standard model, including a right-handed neutrino:

$$
\mathbf{1}_{0}=(\mathcal{V})_{\mathbf{R}} \quad \mathbf{1}_{-1}=e_{\mathbf{R}} \quad \mathbf{2}_{-1 / 2}=\left(\begin{array}{c}
\mathcal{V}_{\mathbf{L}} \\
e_{\mathbf{L}}
\end{array}\right)
$$

(the normalisation is such that the electric charge is $Q=I_{3}+Y$ ). Now for $\mathbf{C P}^{2}$ complex conjugation preserves the chirality and sends $Y_{(2)} \rightarrow \bar{Y}_{(2)}=-Y_{(2)}-3$, so complex conjugation maps (2.8) to

$$
\mathbf{1}_{-1}=e_{\mathbf{R}} \quad \mathbf{1}_{0}=(\mathcal{V})_{\mathbf{R}} \quad \overline{\mathbf{2}}_{-1 / 2}=\left(\begin{array}{c}
e_{\mathbf{L}} \\
\mathcal{V}_{\mathbf{L}}
\end{array}\right)
$$

(for the $\overline{\mathbf{2}}$ the electric charge is $Q=-I_{3}+Y$ and of course the $\overline{\mathbf{2}}$ is equivalent to the 2 under rotation by the Pauli matrix $i \sigma_{2}$ ). The curious conclusion is that, contrary to what one might naïvely expect, complex conjugation does not change the sign of the hypercharge but instead interchanges the electron and neutrino, leaving the electroweak multiplet in (2.8) invariant.

One complete generation of the quark sector of the standard model can be obtained by combining (2.8) with the $S U(3)$ triplet in (2.5), provided the total hypercharge is defined as a particular linear combination of the $\mathbf{C P}^{2}$ and the $\mathbf{C P}^{3}$ charges, $Y=-\frac{1}{4} Y_{(3)}+\frac{1}{3} Y_{(2)}$. Taking the total chirality to be the product of the two individual chiralities, and interpreting positive chirality as right-handed, gives the particle spectrum of the strong sector of the standard model:

$$
(\mathbf{3}, \mathbf{1})_{2 / 3}=u_{\mathbf{R}} \quad(\mathbf{3}, \mathbf{1})_{-1 / 3}=d_{\mathbf{R}} \quad(\mathbf{3}, \mathbf{2})_{1 / 6}=\left(\begin{array}{c}
u_{\mathbf{L}} \\
d_{\mathbf{L}}
\end{array}\right) .
$$

The electroweak sector can be included by combining an $S U(3)$ singlet on $\mathbf{C P}^{3}$, with zero charge and $\nu_{0}=1$, with (2.9):

$$
(\mathbf{1}, \mathbf{1})_{0}=\mathcal{V}_{\mathbf{R}} \quad(\mathbf{1}, \mathbf{1})_{-1}=e_{\mathbf{R}} \quad(\mathbf{1}, \mathbf{2})_{-1 / 2}=\left(\begin{array}{c}
\mathcal{V}_{\mathbf{L}} \\
e_{\mathbf{L}}
\end{array}\right)
$$

Equations (2.10) and (2.11) constitute a single generation of the standard model.

Now observe that the combination $Y=\frac{1}{4} Y_{(3)}-\frac{1}{3} Y_{(2)}$ does simply change sign under complex conjugation, $Y \rightarrow \bar{Y}=-Y$. In addition the $\mathbf{C P}^{3}$ chirality changes while that of $\mathbf{C P}^{2}$ does not so the overall chirality, which is the product of the two, flips. The net result is that complex conjugation of (2.10) and (2.11) does indeed reproduce the anti-particles:

$$
\begin{aligned}
(\mathbf{3}, \mathbf{1})_{2 / 3} & =u_{\mathbf{R}}, \quad(\mathbf{3}, \mathbf{1})_{-1 / 3}=d_{\mathbf{R}}, \quad(\mathbf{3}, \mathbf{2})_{1 / 6}=\left(\begin{array}{c}
u_{\mathbf{L}} \\
d_{\mathbf{L}}
\end{array}\right) \\
\text { c.c. } \quad(\overline{\mathbf{3}}, \mathbf{1})_{-2 / 3} & =(\bar{u})_{\mathbf{L}}, \quad(\overline{\mathbf{3}}, \mathbf{1})_{1 / 3}=(\bar{d})_{\mathbf{L}}, \quad(\overline{\mathbf{3}}, \overline{\mathbf{2}})_{-1 / 6}=\left(\begin{array}{c}
(\bar{u})_{\mathbf{R}} \\
(\bar{d})_{\mathbf{R}}
\end{array}\right)
\end{aligned}
$$


and

$$
\begin{aligned}
(\mathbf{1}, \mathbf{1})_{0} & =\mathcal{V}_{\mathbf{R}}, \quad(\mathbf{1}, \mathbf{1})_{-1}=e_{\mathbf{R}}, \quad(\mathbf{1}, \mathbf{2})_{-1 / 2}=\left(\begin{array}{c}
\mathcal{V}_{\mathbf{L}} \\
e_{\mathbf{L}}
\end{array}\right) \\
\longmapsto \quad(\mathbf{1}, \mathbf{1})_{0} & =(\overline{\mathcal{V}})_{\mathbf{L}}, \quad(\mathbf{1}, \mathbf{1})_{1}=(\bar{e})_{\mathbf{L}}, \quad(\mathbf{1}, \overline{\mathbf{2}})_{1 / 2}=\left(\begin{array}{c}
(\overline{\mathcal{V}})_{\mathbf{R}} \\
(\bar{e})_{\mathbf{R}}
\end{array}\right) .
\end{aligned}
$$

It is interesting that the construction presented here necessarily requires the introduction of a right-handed neutrino, as recent experimental evidence for neutrino oscillations requires just such a state for the simplest explanation of the results [3], [四].

In summary a single complete generation of the standard model, (2.10) and (2.11), arises from a selection of five different bundles, three over $\mathbf{C P}^{2}$ and two over $\mathrm{CP}^{3}$ :

- Two $S U(2)$ singlets on $\mathbf{C P}^{2}$ with $q=0$ and $q=-3$ and an $S U(2)$ doublet with $q=-2$;

- An $S U(3)$ singlet on $\mathbf{C P}^{3}$ with $q=0$ and an $S U(3)$ triplet with $q=-3$.

In terms of the $\operatorname{Spin}^{c}$ structures described in the appendix these correspond to the two bundles

$$
\begin{array}{ll}
\text { on } \mathbf{C P}^{2} & \wedge^{0, *} T \mathbf{C} \mathbf{P}^{2} \otimes\left(\mathbf{1} \oplus L_{(2)}^{3} \oplus\left(F_{(2)} \otimes L_{(2)}^{2}\right)\right) \\
\text { on } \mathbf{C P}^{3} & \wedge^{0, *} T \mathbf{C} \mathbf{P}^{3} \otimes\left(\mathbf{1} \oplus\left(F_{(3)} \otimes L_{(3)}^{3}\right)\right)
\end{array}
$$

where, on $\mathbf{C P}^{2}, L_{(2)}$ is the generating line bundle and $F_{(2)}$ the rank 2 bundle satisfying $L_{(2)} \oplus F_{(2)}=I^{3}$, while, on $\mathbf{C P}^{3}, L_{(3)}$ is the generating line bundle and $F_{(3)}$ the rank 3 bundle satisfying $L_{(3)} \oplus F_{(3)}=I^{4}$, with $I^{3}$ and $I^{4}$ denoting trivial bundles of rank 3 and 4 respectively.

\section{Conclusions}

It has been shown that a single generation of the standard model Fermion spectrum, including a right-handed neutrino, can be obtained from the holonomy groups of $\mathbf{C P}^{2}$ and $\mathbf{C P}^{3}$ by tensoring with appropriate bundles. Physically this means introducing background $S U(3) \times S U(2) \times U(1)$ gauge fields which are topologically non-trivial, containing analogues of monopoles and instantons. A number of questions present themselves.

Firstly there is no obvious sign of three generations. Of course one can obtain more generations by taking copies, but there seems no compelling reason to take three such copies and not some other number. This may be related to the question of what possible rôle the isometry group may play. In the introduction a virtue 
was made of the fact that the construction is not tied down to a specific metric on $\mathbf{C P}^{n}$, but if one introduces the Fubini-Study metric one then has isometry group $S U(n+1)$. On $\mathbf{C P}^{2}$ one has $S U(3)$ and, using this as a horizontal generation group, the fundamental representation would give three generations. But then it is not clear what the rôle of the $S U(4)$ from $\mathbf{C P}^{3}$ would be. Alternatively it is possible to manufacture three copies by including $\mathbf{C P}^{1}$ with $q=2$, giving $\nu_{2}=3$, and then simply ignoring the $U(1)$ charge on $\mathbf{C P}^{1}$. For the moment we have no compelling suggestion as to how the generations might appear and we leave this as an open question.

Secondly there is the question of the extra $U(1)$. The holonomy group of $\mathbf{C P}^{2} \times$ $\mathbf{C P}^{3}$ is $U(3) \times U(2) \cong S U(3) \times S U(2) \times U(1) \times U(1)$ and we have taken a $U(1)$ which is only one particular combination of the two $U(1)$ factors, ignoring the other one. In fact the standard model spectrum has true group $S(U(3) \times U(2))$, [5], and the very fact that it is reproduced here means that the group being used is $S(U(3) \times U(2))$ rather than the full holonomy group $U(3) \times U(2)$. There may be a deeper reason for this, but for the moment we confine ourselves to observing that it works empirically.

How might the construction fit into realistic models? It would certainly seem too naïve to take $\mathbf{C P}^{2} \times \mathbf{C P}^{3}$ as an internal space - it has real dimension 10 which is too large for string theory and simply adding it on to 4-dimensional space-time produces a 14-dimensional space-time which would have anomalies. It may be that one could realise $\mathbf{C P}^{2}$ and $\mathbf{C P}^{3}$ as a brane within a brane in type IIA string theory, which has anti-symmetric tensor fields of rank 2 and 4 in its R-R sector as well as their hodge duals, though IIA string string theory is a non-chiral theory so the Weyl Fermion on $\mathbf{C P}^{3}$ would have to be put in by hand. Any such interpretation would necessarily be rather different to the standard approach, as it would not involve grand unified theories directly. Alternatively a "fuzzy" Kaluza-Klein approach may be of interest where the continuum manifolds of $\mathbf{C P}^{2}$ and $\mathbf{C P}^{3}$ are replaced by non-commutative finite dimensional matrix approximations with a finite number of degrees of freedom [2]. It would then be more appropriate to think of multiple copies of 4-dimensional space-time rather than an internal continuous manifold, somewhat analogous to Connes' non-commutative geometry approach to the standard model with two copies of space-time [6], [7]. Star-products on $\mathbf{C P}^{n}$ were studied in [8] and [9], and the spectrum of the Dirac operator was investigated in [10] and [11]. The smallest vector space that could be used for a non-trivial matrix representation is $n+1$. For $\mathbf{C P}^{2}$ we get 3 , which relates to the discussion of the generation problem above, while $\mathbf{C P}^{2} \times \mathbf{C P}^{3}$ would require $3 \times 4=12$ copies.

While these are all interesting and important problems we leave them open for further work. 


\section{A. The Index Theorem For $\mathrm{CP}^{n}$}

For a general complex manifold $X$ of dimension $n$ the total Chern class is the sum of the individual Chern classes

$$
c(X)=1+c_{1}(X)+c_{2}(X)+\cdots+c_{n}(X) .
$$

In line with common usage we write $c_{k}(X)$ for $c_{k}(T X)$ - the $k^{\text {th }}$ Chern class of the tangent bundle. In particular $c_{n}(X)$ is the top form and the Gauss-Bonnet theorem states that evaluating $c_{n}(X)$ on $X$ gives the Euler characteristic of $X$ : i.e. $c_{n}(X)[X]=\chi(X)$.

For $\mathbf{C P}^{n}$ the Chern classes are all generated by a single 2 dimensional class $x$ [12]

$$
c\left(\mathbf{C P}^{n}\right)=(1+x)^{n+1}=1+(n+1) x+\frac{n(n+1)}{2} x^{2}+\cdots+(n+1) x^{n} .
$$

Note that $x^{n+1}$ is a $(2 n+2)$ dimensional class and so $x^{n+1}=0$ - when $x$ is represented

by a 2 form $\omega$, say, this corresponds to the fact that $\omega^{n+1}=0$ on a $2 n$-dimensional manifold.

The normalisation is such that

$$
\int_{\mathbf{C P}^{n}} \omega^{n}=1
$$

and so, since $c_{n}(X)=(n+1) x^{n} \equiv(n+1) \omega^{n}$, we have

$$
\chi\left(\mathbf{C P}^{n}\right)=n+1
$$

which is the Euler characteristic of $\mathbf{C P}^{n}$ as required by the Gauss-Bonnet theorem. The form $\omega$ is the curvature of a line bundle $\bar{L}$ whose complex conjugate $L$ we shall refer to as the generating line bundle over $\mathbf{C P}^{n}$ with Chern class

$$
c(L)=1-x .
$$

Another line bundle that will be important is the canonical line bundle $K$ which is the maximum exterior power of the cotangent bundle $T^{*} X$ : i.e.

$$
K=\wedge^{n} T^{*} X
$$

$K$ has Chern class given by

$$
c(K)=1-(n+1) x .
$$

The minus sign appears because

$$
c_{1}(K)=c_{1}\left(T^{*} X\right)=-c_{1}(T X) .
$$


We see that $K$ is a power of the generating line bundle $L$; in fact we have

$$
K=L^{n+1} .
$$

The existence of global spinors is determined by the second Stiefel-Whitney class $w_{2}$, which, on a complex manifold $X$, can be obtained from $c_{1}$ by reducing mod 2 . One has

$$
w_{2}(X)=c_{1}(X) \bmod 2 \quad(X \text { complex }) .
$$

For $X=\mathbf{C P}^{n}$ we see that

$$
\begin{aligned}
w_{2}(X) & =(n+1) x \bmod 2, \quad X=\mathbf{C P}^{n} \\
& = \begin{cases}0, & \text { if } n \text { is odd } \\
\neq 0, & \text { if } n \text { is even. }\end{cases}
\end{aligned}
$$

Hence $\mathbf{C P}^{n}$ admits globally defined spinors for odd $n$ but not for even $n$.

When $n$ is even it is still possible to define what is called a $\operatorname{Spin}^{c}$ structure and this gives a more general kind of spinor which comes accompanied by a line bundle and hence a possible $U(1)$ connection.

Moreover, when, as is the case here, $X$ is a complex manifold both spin structures and Spin $^{c}$ structures have a concrete form in terms of differential forms which goes as follows: consider the bundle $\wedge^{0, *} T X$ of all forms of type $(0, k)$-i.e. anti-holomorphic $k$ forms - so we have

$$
\wedge^{0, *} T X=\bigoplus_{k} \wedge^{0, k} \overline{T^{*} X} .
$$

Then this is the $\operatorname{Spin}^{c}$ bundle $S^{c}(X)$ i.e.

$$
S^{c}(X)=\wedge^{0, *} T X .
$$

Hence, for a complex Kähler manifold, generalised spinors are $(0, k)$ forms and are sections of $S^{c}(X)$; the Dirac operator is then the operator $\bar{\partial}+\bar{\partial}^{*}$. Now if $X$ is not just a $S p i n^{c}$ manifold but also a spin manifold then it has a bundle $S(X)$ of true spinors given by

$$
\begin{aligned}
S(X) & =S^{c}(X) \otimes K^{1 / 2} \\
\Rightarrow S^{c}(X) & =S(X) \otimes K^{-1 / 2} .
\end{aligned}
$$

Now the point is that the LHS of A.16 always exists but the two bundles $S(X)$ and $K^{-1 / 2}$ on the RHS only exist separately when $X$ is a spin manifold. One can still think of sections of $S^{c}(X)$ as being given 'locally' by sections of $S(X) \otimes K^{-1 / 2}$-hence the interpretation of $\operatorname{Spin}^{c}$ structures as being (locally) a spinor with an attendant $U(1)$ gauge connection.

Specialising to the case

$$
X=\mathbf{C P}^{n}
$$


we see that $S^{c}\left(\mathbf{C P}^{n}\right)=\wedge^{0, *} T \mathbf{C P}^{n}$ exists for all $n$ but $S\left(\mathbf{C P}^{n}\right)$ and $K^{-1 / 2}$ exist only for odd $n$.

We can construct more general $U(1)$ bundles by adding more integral powers of $L$ giving

$$
\begin{aligned}
& S^{c}(X) \otimes L^{-q} \\
= & \wedge^{0, *} T X \otimes L^{-q}, \quad X=\mathbf{C P}^{n} \\
= & S(X) \otimes L^{p}, \quad \text { where } p=-q-\frac{n+1}{2}
\end{aligned}
$$

and we have used the fact that $K=L^{n+1}$; note too that $p$ is an integer for $n$ odd and a half-integer for $n$ even (the minus sign in the exponent $L^{-q}$ is for later convenience).

In physics language tensoring with powers of $L$ means introducing a $U(1)$ gauge field and $q$ will be related to the $U(1)$ charge. It is analogous to a monopole charge which is evaluated by integrating the Chern character $\operatorname{ch}\left(L^{-q}\right)$ over a non-trivial 2-cycle (a 2-sphere) in $\mathbf{C P}^{n}$. Our conventions are such that

$$
\int_{S^{2}} \operatorname{ch}(L)=\int_{S^{2}} c_{1}(L)=-1
$$

SO

$$
\int_{S^{2}} \operatorname{ch}\left(L^{-q}\right)=-q \int_{S^{2}} c_{1}(L)=q
$$

For a given $p$ the net number of zero modes of the Dirac operator is given by the index theorem as $\nu$ where

$$
\nu=\int_{X} \operatorname{ch}\left(L^{p}\right) \hat{A}(X), \quad X=\mathbf{C P}^{n} .
$$

This equation can be written solely in terms of the Kähler 2-form $\omega$ : since the $\hat{A}$-genus and the Chern character for $\mathbf{C P}^{n}$ are given by

$$
\begin{aligned}
\hat{A}(X) & =\left(\frac{\omega}{2 \sinh (\omega / 2)}\right)^{n+1} \quad \text { and } \quad \operatorname{ch}\left(L^{p}\right)=e^{-p \omega} \\
\Rightarrow \operatorname{ch}\left(L^{p}\right) \hat{A}(X) & =e^{-p \omega}\left(\frac{\omega}{e^{\omega / 2}-e^{-\omega / 2}}\right)^{n+1}, \quad X=\mathbf{C P}^{n} \\
& =e^{q \omega}\left(\frac{\omega}{1-e^{-\omega}}\right)^{n+1} \quad \text { since } \quad p=-q-\frac{n+1}{2} .
\end{aligned}
$$

Since $\int_{\mathbf{C P}^{n}} \omega^{n}=1$ the index, which we shall denote by $\nu_{q}$, is the coefficient of the $\omega^{n}$ term. This coefficient can be evaluated by integration around a small contour enclosing the origin in the complex $z$-plane giving

$$
\nu_{q}=\frac{1}{2 \pi i} \oint \frac{e^{q z} d z}{z^{n+1}}\left(\frac{z}{1-e^{-z}}\right)^{n+1} \text {. }
$$


A change of variable to $t=1-e^{-z}$ yields the answer

$$
\nu_{q}=\left(\begin{array}{c}
q+n \\
n
\end{array}\right)=\frac{1}{n !}(q+1) \cdots(q+n) .
$$

This formula works for both even and odd $n$ and for any integral $q$, either positive or negative. The index for a Fermion coupled to a $U(1)$ gauge field on $\mathbf{C P}^{2}$ was derived in 13.

Another bundle that is of interest when $n \geq 2$ is the rank $n$ vector bundle $F$ that is inverse to the generating line bundle $L$ in the sense that $F \oplus L=I^{n+1}$, with $I^{n+1}$ the trivial $n+1$ bundle. Since $F \oplus L$ is trivial we find that

$$
\begin{aligned}
\operatorname{ch}(F \oplus L) & =\operatorname{ch}(F)+\operatorname{ch}(L)=n+1 \\
\Rightarrow \operatorname{ch}(F) & =n+1-\operatorname{ch}(L) .
\end{aligned}
$$

A second property of the Chern character that will be used below is

$$
\begin{aligned}
\operatorname{ch}(F \otimes L) & =\operatorname{ch}(F) \operatorname{ch}(L) \\
\Rightarrow \operatorname{ch}\left(F \otimes L^{p}\right) & =(n+1) \operatorname{ch}\left(L^{p}\right)-\operatorname{ch}\left(L^{p+1}\right)
\end{aligned}
$$

The bundle $F$ has structure group $U(n)$ and supports a curvature 2 form of a necessarily topologically non-trivial Yang-Mills gauge field on $\mathbf{C P}^{n}$.

This provides sufficient information to work out the zero mode structure of the Dirac operator for spinors transforming under $S U(n) \times U(1)$, in the fundamental representation of $S U(n)$ and in the background gauge field of $F$. More generally we can tensor $F$ with powers of $L$ and determine the zero mode structure of the Dirac operator for a spinor on $\mathbf{C P}^{n}$, in the fundamental representation of $S U(n)$ and a $U(1)$ charge in the background field of $F \otimes L^{p}$. The Dirac index is then denoted by $\nu_{q, \mathbf{n}}$ where

$$
\nu_{q, \mathbf{n}}=\int_{\mathbf{C P}^{n}} \operatorname{ch}(F) \operatorname{ch}\left(L^{p}\right) \hat{A}\left(\mathbf{C P}^{n}\right)=(n+1) \nu_{q}-\nu_{q-1},
$$

where equation ( $\mathrm{A} .32$ ) has been used.

Using (A.28) in (A.33) the index for spinors transforming under the fundamental representation of $S U(n)$ is

$$
\nu_{q, \mathbf{n}}=(q+n+1)\left(\begin{array}{c}
q+n-1 \\
n-1
\end{array}\right)=\frac{(q+n+1)(q+1) \cdots(q+(n-1))}{(n-1) !} .
$$

The presence of $F$ affects the $U(1)$ charge because

$$
\int_{S^{2}} \operatorname{ch}(F) \operatorname{ch}\left(L^{-q}\right)=\int_{S^{2}}\left(n-c_{1}(L)+\cdots\right)\left(1-q c_{1}(L)+\cdots\right)=n q+1 .
$$

Since the Chern character involves a trace, the $U(1)$ generator in the $n \times n$ representation is $\left(q+\frac{1}{n}\right) \mathbf{1}$, where $\mathbf{1}$ is the $n \times n$ identity matrix, and the $U(1)$ charge is therefore $\left(q+\frac{1}{n}\right)$. 
As different $n$ 's are used in the text, when confusion is possible, we shall distinguish the $U(1)$ charges for different $\mathbf{C P}^{n}$ 's with a bracketed subscript $n$, as $Y_{(n)}=q+\frac{1}{n}$, and the different bundles likewise, as $L_{(n)}$ and $F_{(n)}$.

Equations (A.28) and (A.34) are the important results of this analysis. An immediate consequence is that the index vanishes for $S U(n)$ singlets when $q=-1, \cdots,-n$. For Fermions in the fundamental representation of $S U(n)$ the index vanishes for $q=-1, \cdots,-(n-1)$ and $q=-(n+1)$. Generically there are no zero modes for these charges, but even when there are they occur in pairs of opposite chirality.

Two further observations, which will be most important in the analysis in the text, are

- Singlets of $S U(n)$ with zero charge have index $\nu_{0}=1$, while singlets with charge $-(n+1)$ have index $\nu_{-(n+1)}=(-1)^{n}$.

- In the fundamental representation of $S U(n)$ Fermions coupled to $q=-n$ have charge $-n+\frac{1}{n}$ and index $\nu_{-n, \mathbf{n}}=(-1)^{n+1}$.

Finally we evaluate the index for spinors in the complex conjugate representationsthis is relevant to the CPT theorem. For singlets, since $\bar{L}=L^{-1}$, taking the complex conjugate changes the sign of $p$ : one has $p \mapsto \bar{p}=-p$, and $q \mapsto \bar{q}=-q-(n+1)$, giving

$$
\nu_{\bar{q}}=\frac{1}{n !}(\bar{q}+1) \cdots(\bar{q}+n)=(-1)^{n} \nu_{q}
$$

Note that the $U(1)$ charge is $\bar{q}=-q-(n+1)$, and not just $-q$, so $\bar{Y}_{(n)}=-Y_{(n)}-$ $(n+1)$.

For spinors in the complex conjugate fundamental representation $\overline{\mathbf{n}}$ of $S U(n)$ one has, using $\bar{F} \oplus \bar{L}=I^{n+1}$,

$$
\begin{aligned}
\operatorname{ch}(\bar{F}) & =n+1-e^{-c_{1}(L)} \\
\Rightarrow \quad \nu_{\bar{q}, \overline{\mathbf{n}}} & =(n+1) \nu_{\bar{q}}-\nu_{\bar{q}+1} .
\end{aligned}
$$

This implies that

$$
\nu_{\bar{q}, \overline{\mathbf{n}}}=\frac{\bar{q}(\bar{q}+2) \cdots(\bar{q}+n)}{(n-1) !}=(-1)^{n} \nu_{q, \mathbf{n}}
$$

The $U(1)$ charge for $\overline{\mathbf{n}}$ is calculated from

$$
\begin{aligned}
\int_{S^{2}} \operatorname{ch}(\bar{F}) \operatorname{ch}\left(L^{-\bar{q}}\right) & =\int_{S^{2}}\left(n+c_{1}(L)+\cdots\right)\left(1-\bar{q} c_{1}(L)+\cdots\right) \\
& =n \bar{q}-1
\end{aligned}
$$

so, dividing by the rank of the matrices as before, the charge is $\bar{q}-\frac{1}{n}=-(q+n+1)-\frac{1}{n}$ so again $\bar{Y}_{(n)}=-Y_{(n)}-(n+1)$. To summarise the net effect of complex conjugation is to preserve the chiralities if $n$ is even and flip them if $n$ is odd and map the charges to $Y_{(n)} \mapsto \bar{Y}_{(n)}=-Y_{(n)}-(n+1)$. 


\section{References}

[1] E. Witten, in Proceedings of the 1983 Shelter Island Conference on Quantum Field Theory and the Fundamental Problems of Physics, Ed. R. Jackiw, N.N. Khuri, S. Weinberg and E. Witten, MIT Press, (1985)

[2] B. Dolan and C. Nash, Chiral Fermions and Spin ${ }^{c}$ Structures form Matrix Approximations to Manifolds, hep-th/0207007

[3] S. Fukuda et al. Super-Kamiokande collaboration Phys. Rev. Lett. 86, (2001), 5651, hep-ex/0103032; Phys. Rev. Lett. 86, (2001), 5656, hep-ex/0103033

[4] Q.R. Ahmed et at. SNO collaboration Phys. Rev. Lett. 87 (2001) 071301;

nucl-ex/0204008; nucl-ex/0204009

[5] L. O'Raifeartaigh, Group Structure of Gauge Theories, (1987), CUP

[6] A. Connes and J. Lott Nucl. Phys. B (Proc. Suppl.) 18, (1990), 29

[7] Non-commutative Geometry A. Connes, (1994), Academic Press

[8] M. Bordemann, M. Brischle, C. Emmrich and S. Waldmann, J. Math. Phys. 37, (1996), 6311, q-alg/9512019; Lett. Math. Phys. 36, (1996), 357, q-alg/9503004

[9] A.P. Balachandran, Brian P. Dolan, J. Lee, X. Martin, Denjoe O'Connor, Fuzzy Complex Projective Spaces and their Star-products, to appear in J. Geom. Phys. hep-th/0107099

[10] H. Grosse and A. Strohmaier, Lett. Math. Phys. 48, 163, (1999) hep-th/9902138

[11] G. Alexanian, A.P. Balachandran, G. Immirzi and B. Ydri J. Geom. Phys. 42 (2002), 28, hep-th/0103023

[12] R. Bott and L.W. Tu Differential Forms in Algebraic Topology, Springer, Graduate Texts in Mathematics 82 (1982)

[13] S.W. Hawking and C. Pope, Phys. Lett. 73B, 42, (1978) 